\section{Duodenal Web with Trichobezoar: An Unusual Presentation}

Duodenal atresia and duodenal web cause upper gastrointestinal obstruction and usually present in neonatal age soon after birth [1]. However, delayed presentation has been documented in literature in first few months or years of life related to partial obstruction. [2]. Here we report duodenal web presenting in the third year of life associated with trichobezoar in the duodenum.

A 2-year-old girl with Down syndrome presented with complaints of recurrent vomiting of 2-3 months. The vomiting was non-bilious in nature and it contained 'cherry seeds' eaten about 3-4 months before. The child had no history of abdominal distension, blood in vomitus or bowel complaints. The child was well hydrated, afebrile, and with no previous complaints. Abdominal examination showed non-distended abdomen. No definite lump or tenderness was palpable. There was no free fluid and bowel sounds were normal. $X$-ray of abdomen revealed 'double bubble' with paucity of distal gas. A contrast study was done using water-soluble contrast agent, which showed hugely distended stomach with delayed drainage and normal small bowel. She was explored through supra-umbilical transverse incision. A hugely dilated stomach was identified. The second part of duodenum had a windsock deformity. Duodenotomy revealed a pre-ampullary web with trichobezoar obstructing the lumen. The gastric outlet was normal. The child was managed by duodenoduodenostomy. She remained well in post-operative period and is well on follow-up after 3 months.

Duodenal atresia or duodenal web may be identified on antenatal ultrasound or usually presents in first week of life with recurrent vomiting. The condition can be picked up on plain x-ray of abdomen showing 'double-bubble' appearance. Duodenal web; however, may present late due to partial obstruction [1]. Duodenal atresia and duodenal web are caused by abnormal duodenal development at 6-8 weeks of gestation, and are known to be associated with Down syndrome [1].

Trichobezoar is a condition where a collection of hairs form a mass that does not pass into the intestine and causes obstruction. Usually the trichobezoar occurs in the stomach, and it may extend into the intestine as a tail causing Rapunzel syndrome [3]. The trichobezoar occurs more commonly in persons with psychiatric diseases with trichotillomania. Although no behavior of eating hair was noted by parents, the same may be present/ have happened accidentally due to intellectual deficit or inadequate supervision by parents. The cherry seeds reported in history may have precipitated the obstruction either by themselves or by acting as a nidus for the trichobezoar. Trichobezoar is usually diagnosed on ultrasound or CT scan of abdomen, and managed by retrieval through laparotomy or laparoscopy [4].

A similar case has been reported in the French literature [5]. The association of duodenal atresia and Down syndrome helped us in suspecting the duodenal atresia. Although phytobezoars have been reported in early life, the incidence of trichobezoar in third year of life is rare. For a child with Down syndrome and recurrent vomiting, the differential for duodenal atresia should be high on the list and needs to be evaluated and managed promptly.

\section{REFERENCES}

ViVek Manchanda ${ }^{1 *}$, Shekhar BisWas ${ }^{2}$, ANIRUdh GhaI ${ }^{2}$ AND SANJAY GoYal ${ }^{3}$ Departments of ${ }^{1}$ Paediatric Surgery, ${ }^{2}$ Paediatrics and ${ }^{3}$ Radiodiagnosis, Jaipur Golden Hospital, New Delhi, India. *vivek_7477@hotmail.com

1. Ibrahim IA, El Tayab AMA. Congenital duodenal stenosis: Early and late presentation. Med $J$ Cairo Univ. 2013;81:609-17.

2. Guo X, Yu Y, Wang M, Qin R. Case report of a congenital duodenal transverse septum causing partial obstruction. Medicine (Baltimore). 2017;96:e7093.

3. Wang Z, Cao F, Liu D, Fang Y, Li F. The diagnosis and treatment of Rapunzel syndrome. Acta Radiol Open. 2016;5:2058460115627660.

4. Tudor EC, Clark MC. Laparoscopic-assisted removal of gastric trichobezoar: A novel technique to reduce operative complications and time. J Pediatr Surg. 2013;48:e13-5.

5. Chaouachi B, Daghfous S, Ben Salah S, Hammou A, Gharib NA, Saied H. [A case of trichobezoar associated with a duodenal diaphragm]. Ann Pediatr (Paris). 1988;35:205-8 [French].

\section{CASPR2-Mediated Autoimmune Encephalitis in a Toddler}

$\mathrm{A}$ utoimmune limbic encephalitis commonly presents in adults, frequently associated with Leucine-rich glioma inactivated protein-1 (LGI1) and Contactin associated protein-2 (CASPR2) antibodies, and is uncommon in children. Here we report a case of CASPR2 mediated autoimmune limbic encephalitis in a toddler.
A 19-month-old girl, born to consanguineously married parents, who was developing typically, presented with excessive irritabilityand decreased sleep for the past 40-50 days.Child had received diphtheria-pertussis-tetanus(DPT) booster dose 50 days back. Initially the symptoms were attributed to vaccination, but irritability kept gradually increasing. Child gradually regressed in language and cognition. She was not able to speak and on admission was able to only coo.There was behavioural change in the child in the form of new onset head banging, throwing of previously favourite toys and was avoiding going to the mother.Child was not mingling well 